\title{
Factor Affecting Short Term Load Forecasting
}

\author{
Muhammad Usman Fahad and Naeem Arbab
}

\begin{abstract}
The basic objective of short term load forecasting is to predict the near future load for example next hour load prediction or next day load prediction etc. The total system load is the load seen at the generating end of the power system, which includes the sum of all types of loads connected to the system plus the losses. To design efficient and accurate forecasting model one must have good understanding of the characteristics of the system. There are various factors which influence the behavior of the consumer load and also impact the total losses in transmission lines. These factors can be categorized as Time factor, weather, economy and random disturbances. In this research paper these factors and their impact on consumption of electric power and their significance in short term load forecasting is evaluated.
\end{abstract}

Index Terms-ANN, factors of STLF, load forecasting, load prediction.

\section{TIME FACTOR}

Time is the most important factor in short term load forecasting because its impact on consumer load is highest. From observing load curve of several different grid stations it is found that the load curve has "time of the day" property, also it has "day of week", "week of month" and "month of season" property. This means that load curve is periodic in nature. Fig. 1(a)-Fig. 1(c) shows the load curve of 3 Fridays of the April 2012.As can be seen in Fig. 1(a)-Fig. 1(c) that although the average load is different because of the difference of temperature during each day but the pattern of the load is nearly same. In each curve there arises three peaks First peak at 8 o clock, $2^{\text {nd }}$ peak at 12:30 pm, And third at 8 $\mathrm{pm}$ and in curves first peak is lowest $2^{\text {nd }}$ a bit taller and third the highest. This shows periodicity in terms of day of the week.

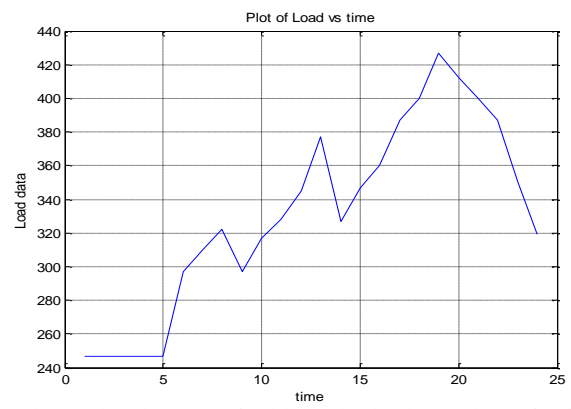

Fig. 1(a). A 24 hours load curve of Abbottabad grid station of Friday $6^{\text {th }}$ April 2012.

Manuscript received August 17, 2013; revised December 16, 2013. This work was carried out as a final year project for the award of MS degree in electrical engineering.

Muhammad Usman Fahad is with the University of Engineering and Technology, Peshawar, Pakistan (e-mail: usman_fahad4@ hotmail.com).

Naeem Arbab is with the Electrical Engineering Department of University of Engineering and Technology, Peshawar, Pakistan (e-mail: mnarbab01@yahoo.com).

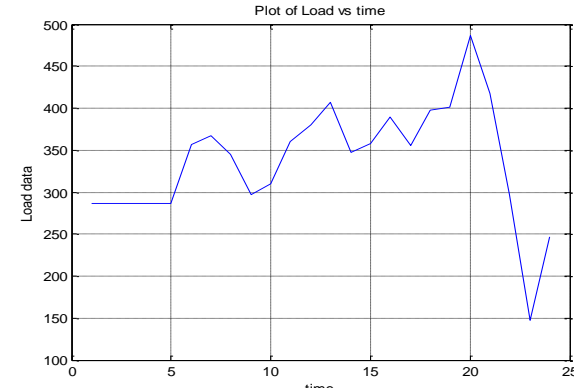

Fig. 1(b). A 24 hours load curve of Abbottabad grid station of Friday $13^{\text {th }}$ April 2012.

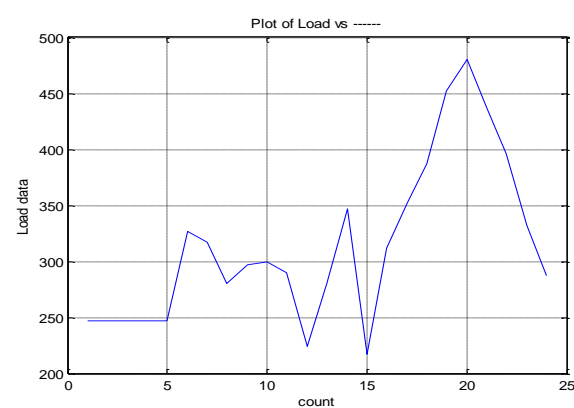

Fig. 1(c). A 24 hours load curve of Abbottabad grid station of Friday $20^{\text {th }}$ April 2012.

From the observation of daily load curve of any grid station it can be seen that load variation follows certain rules with the "time point" of the day.

In Fig. 2 hourly load curves is shown for 24 hours of a day in winter season. The interval length is one hour, so there are 24 intervals in the figure. It can be seen in figure that the load is low and stable from 0 am to 6 am (0am means 12 o clock at night), the load start rising at 7 o clock till 9 am and then it is flattened till $12 \mathrm{am}$ and after that it descends till $5 \mathrm{pm}(17: 00$ in figure) and after $5 \mathrm{pm}$ it start rising till $8 \mathrm{pm}$ (19:00), after 8 pm load gradually decrease again until the end of the day.

It can be seen in Fig. 2 that maximum demand occurs at $8 \mathrm{pm}$, and minimum load demand occurs after mid night. So if we closely observe this load curve it can be seen that load demand reflects the consumer's daily life style. At mid night everyone is sleeping so there is no need of lighting or heating the house, so load becomes least. Similarly at $8 \mathrm{pm}$ everyone is at home watching TV, sitting beside heater etc, so load is highest at that part of the day.

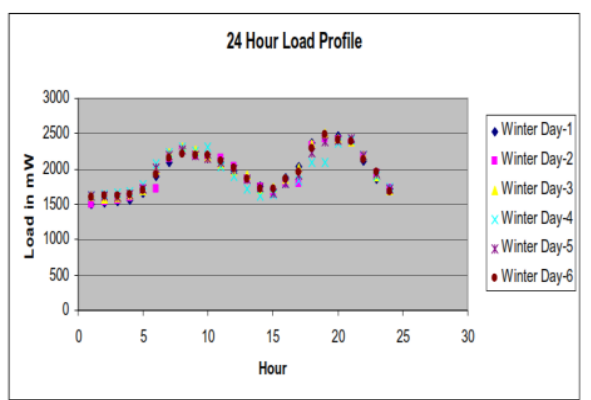

Fig. 2. A 24 hours load curve of Abbottabad grid station during week of winter. 
The people's daily life activities can be classified in to three parts

- Working Time

- Leisure Time

- Sleeping Time

In Fig. 2 the load curve has two peaks one in the working time and one in the leisure time, while at sleeping time load curve shows least value.

The rule of load variation discussed above is not the only rule. There are certainly other rules of load variation with time. For example weekend and national holiday load is observed to be lower than the week day loads due to the absence of factory and office loads.

The start of semester of universities or school year also has the significant impact on load consumption and thus changes the load profile. Similarly day light saving can also decrease the average daily load also it shift the peaks from one time spot to the other.

It is noticed from the collected load data that the load curve is periodic. This periodicity of load occurs not only in the daily load but periodicity is present in weekly, monthly and seasonal and yearly load curves. This is very important property of load curve because by taking periodicity of load in to account we can predict or forecast load more effectively.

\section{ECONOMIC FACTOR}

Since electricity nowadays became people's daily life necessity so it has turned to be a commodity. Thus economy of the state has also an impact on the usage of electricity. Economic factor has more importance in long term forecasting [1], but it also can impact the load curve for short term load forecasting.

For example the daily load curve of developed countries shows different patterns as compared to the daily loads curve of under developed countries. Huge peak is observed in daily load curve for developed countries after 11:00 am till 4:00 pm due to large industrial activity, where as peak for under developed countries will arise after 6:00 pm. Thus for load forecasting we must consider the country's economic situation (GDP), and also must have glance on the industrial development of the country for long term load forecasting [1].

Price of electricity and the people's buying capability also have impact on the usage of electricity, hence more expensive the electricity is, less it will be used by the domestic consumers. For example using Air conditioner is non affordable for the most population of Pakistan. Thus price of electricity also affect the daily load curve [1].

Time of use pricing can alter the duration and the time of occurrence of peak load. In some countries electricity is cheaper at night than at day. Thus by doing that night valley disappears because people tend to use electricity for heat storage equipments at night and during day use stored heat for warming the rooms and homes etc. Therefore time of use pricing can make domestic as well as industrial consumers to adjust their load and thus helps in peak shaving and filling of the night valley.

Therefore for short term load forecasting time of use pricing is yet another factor that can alter not only the daily load curve pattern but also can reduce the average daily load and hence should be considered for short term load forecasting as an important factor.

Hence economic factors such as price of electricity, management of load and degree of industrialization have a significant impact on system average load and system maximum demand.

\section{WEATHER}

Weather is the most important independent variable for load forecasting. The effect of weather is most prominent for domestic and agricultural consumers, but it can also alter the load profile of industrial consumers.

Load forecasting models use weather forecast and other factors to predict the future load, thus to minimize the operational cost.

Weather is often cited as the tipping point that can cause unreliability in the system by decreasing the efficient supply of power. Unpredicted sea breeze, after moon thunderstorms, back door fronts is some of the environmental factors that can decrease the temperature and thus causing overestimated load forecast [2]. Thus we are producing more power than required.

Similarly under forecasting of summer temperature can cause load swing that may cost 20,000 rupees per mega watt hour [3].

Temperature can also alter the conductivity of the transmission lines. Thus temperature can affect the overall carrying capability of the transmission lines. High temperature can increase not only the resistance of the transmission lines, but also it can alter the reactance of line, due to temperature induced expansion of the length of transmission line.

The weather factor include the following [4], [5]

- Temperature

- Humidity

- Precipitation

- Wind speed and wind chill index

- Cloud cover and light intensity

Let us define all these factors and then we will explain their effect on short term load forecasting.

\section{A. Temperature}

Temperature is the measure of average kinetic energy of the atoms or molecules of an object.

Or it can be defined as

"The measure of degree of hotness or coldness of a body"

The results of the D Paravan [6], shows that there is a high positive correlation between temperature and load during summer season and there is a negative correlation between temperature and load during winter.

This means that during summer increase in temperature will result in increase in load and decrease in temperature will result in decrease in not only average daily load but also will lower the peak demand. But in winter the opposite of the above will happen, during winter decrease in per degree temperature will results in increase of electric load. For this negative correlation Pardo et al. proposed a transfer function intervention model to predict the electricity demand in the hot and cold days [7].

This is because during summer increase in temperature 
affect the people's feeling of comfort and consumers will use electricity for cooling purpose (more AC and fans will be used), whereas in winter electricity is used for heating purposes. Hence in winter there is negative or inverse relation between temperature and load consumption.

My research showed that temperature; electric load correlation is positive for any temperature higher than $25^{\circ} \mathrm{C}$ and temperature, electric load correlation is negative for any temperature lower than $20^{\circ} \mathrm{C}$. In between 20 and $25^{\circ} \mathrm{C}$ the temperature factor has least significance. So the relation between temperature and load consumption is very complex in nature and cannot be analyzed with ordinary mathematical models [8].

\section{B. Humidity}

Humidity is a term used for the amount of water vapors in air.

Formally humid air was called not just the moist air but was referred as the mixture of water vapors and other constituents of air and humidity was defined in terms of water contents of this mixture called the absolute humidity [9]. In everyday life it is called relative humidity and is expressed in percentage.

It is common observation that humidity can increase apparent temperature while it has no effect on the real temperature. This means humidity can make a $30^{\circ} \mathrm{C}$ temperature to be felt say $35^{\circ} \mathrm{C}$. But according to the researchers at Pen state, high humidity can make it feel colder when the temperature is below $53^{\circ} \mathrm{F}$ and it can make feel warmer when the temperature is higher than $53 \mathrm{~F}$

Humans are sensitive to humidity because the mechanism used to regulate the body temperature is evaporative cooling. At high humid atmosphere the rate of evaporation through skin (perspiration) is lower than it would be under normal conditions. Since human perceives rate of transfer of heat rather than temperature itself, so we feel warmer at high humid conditions.

Thus humidity can increase the feeling of the severity of temperature and make people to use more cooling appliances therefore due to this fact daily load curve will show high value during humid day. For example humidity is about $100 \%$ during month of august (rainy season) and it is practically observed from the past data collected from WAPDA grid station abbottabad (Pakistan) that average monthly load is highest during the month of august.

Although humidity has no effect on real temperature but it can intensify the severity of hot climate. So it is concluded from the above observation that for the prediction of daily load of domestic consumers we must consider apparent temperature instead of real temperature. But if we are dealing with an area having all types of consumers from industrial to agricultural and domestic "temperature humidity index" can be employed as the affecting factor for load forecasting.

\section{Precipitation}

It can be defined as

"The quantity of water fallen on earth at a specific place in a specific period of time."

Or better and more accurate definition of precipitation may be,

"The amount of rain, snow or hail fallen at a specific place within a specific period of time."
It is expressed in inches or centimeter of water.

Precipitation can affect load consumption directly and indirectly.

\section{1) Direct effect}

Heavy rain or snow can make people to stay home and it can cause darkness also. So due to the fact that people will be forced to stay in door they will consume more electricity for lighting purpose and for entertainment appliances.

\section{2) Indirect effect}

By indirect effect we mean that heavy rain or snow can decrease the temperature thus may have positive or negative effect on load consumption. By negative we mean load consumption will increase and by positive we mean decrease in load consumption [10]

In summer effect of precipitation is positive because temperature will decrease and become pleasant so less AC and other cooling appliances will be used by domestic consumers. In Abbottabad town rain in summer even in the month of June can often make the weather so pleasant that people do not feel need to use even fans, so due to precipitation load will decrease dramatically in summer and hence precipitation is considered an important factor for next hour load forecasting(very short term load forecasting).

In winter season the effect of precipitation is negative because it can decrease the temperature and can further intensify the severity of cold weather. Therefore due to extensive use of electric heater during rain a sudden peak will arise [11], [12].

Snow in winter or even rain in winter decrease temperature reasonably and also make people to stay in door causing more power used for heating purpose and lighting the room.

So for short term load forecasting precipitation factor must be considered to accurately predict the load and if not considered the predicted load may be wrong by huge margin thus causing huge loss for the power company (in case of over generation in summer) or may result in load shedding (in case of under generation).

\section{Wind Speed and Wind Chill Index}

Wind speed can be defined as

"The measure of the motion of air with respect to the surface of the earth covering a unit distance in unit time"

Wind speed can affect weather forecast, it is now measured with anemometer but it can also be measured using the older Beaufort scale which is based on people's observation on specially defined effects of wind.

Wind direction and speed is affected by three main factors

- Fractional forces e.g. forest, mountains and buildings etc.

- Temperature gradient also sometimes referred as pressure gradient

- Earth rotation known as Coriolis Effect.

\section{1) Effect of wind speed on load consumption}

At low humidity rates the speed of wind lowers the apparent temperature and increases the rate of evaporation of perspiration from the human body therefore it gives the cooling effect.

Thus during a windy day of summer the consumption of electricity will be lower because lesser cooling appliances will be used. 
If we have mixed generation network i.e. we have hydrogenation plus renewable energy resources generator including wind power generators then the output power of wind generators will be high during a windy day.

Under these conditions if our model is forecasting the load demand of generating stations then we must consider wind speed as important factor because instead of over generation by the thermal generators, the short fall can be compensated by the increase in power generation of wind turbines, because electricity generated by wind turbines is economical then the same generated by thermal or even by hydropower plants.

Similarly since apparent temperature is highly dependent on wind speed therefore during a windy day, domestic load consumption will be low, so load forecasting model employed even for distribution feeders must also consider the wind speed as an affecting factor that can not only decrease load consumption(summer) but also can increase the load consumption. This increase in load consumption is further described under the heading of wind chill index.

\section{2) Wind chill index}

Wind chill factor is the felt air temperature on exposed skin due to wind.

Wind chill temperature is always less than the air temperature and is undefined at temperature above $10^{\circ} \mathrm{C}$.

Any hot body makes the surrounding air warmer. The warmer air surrounding the body then acts as insulator preventing the further heat loss. But if the wind blows then the colder air takes the place of the warmer air thus causing further heat loss. The speed of the heat loss is directly proportional to the wind speed. Greater the wind speed higher will be the heat loss. The above phenomenon is called wind chill.

Thus the effect of wind chill is to reduce the temperature of the warmer bodies to the ambient temperature more quickly. Note however that no matter how fast the wind is blowing it cannot reduce the temperature of a body below the ambient temperature.

But human feels the temperature far below the ambient temperature because human body tries to maintain its temperature at $37^{\circ} \mathrm{C}$ thus causing more heat loss and increasing the risk of frost bite and hypothermia.

Therefore in winter season load consumption on a windy day will be high [13]. Thus wind speed can also affect the load consumption. And wind chill index may also be considered as an input to the load forecasting model.

The formula for WCI is [14]

$$
\mathrm{WCI}=(10 \sqrt{V}-V+10.5) \times(33-T)
$$

where $V$ is the velocity of wind in $\mathrm{m} / \mathrm{s}$, WCI is wind chill index and $T$ is air temperature in ${ }^{\circ} C$.

A new and improved formula developed by US and Canada [14] which has higher accuracy is

$$
\text { TWCI }=13.12+0.6215 T-11.37 V^{0.16}+0.3965 T \times V^{0.16}(2)
$$

where TWCI is the wind chill temperature, $V$ is the velocity of wind in $\mathrm{m} / \mathrm{s}$, and $T$ is air temperature.

In Fig. 3 the effect of wind speed on felt air temperature is explained. The Temperature is supposed to be $15^{\circ} \mathrm{C}$ and the wind chill temperature is plotted against the wind speed by both new and by old formula.

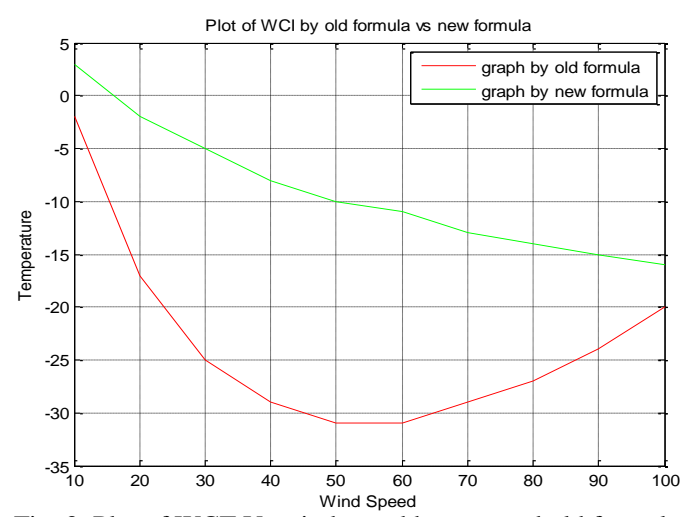

Fig. 3. Plot of WCT Vs wind speed by new and old formula

\section{E. Cloud Cover and Light Intensity}

The effect of cloud cover on load consumption depends upon the height and thickness of clouds and the time of the day.

The high and thin cloud has very little impact on temperature especially at night so it can be ignored for Short term load forecasting. The more important type is low and thick cloud that has major impact on air temperature and is discussed here.

The effect of cloud on temperature is the net effect of the following three things [15].

- Green house effect of clouds

- Reflection of the sun light from top of cloud

- Reflection of the thermal radiation of earth from cloud back to the surface.

Since the effect of cloud on load consumption depends upon time of the day. At night there is no sun light so cooling effect of cloud cover is zero. So at night cloud has green house effect as it traps heat in to the atmosphere, and it also reflects the thermal radiation from earth back to the surface and hence has net warming effect. Thus cloud cover at night can increase temperature significantly also the next day temperature will be high. So during summer next day load will be very high.

The green house effect of the cloud reflects only $50 \%$ of the outgoing radiation but on the other hand reflection of thermal radiation is about $90 \%$. So at night thermal reflection of cloud plays more important role in warming up the atmosphere then the green house effect.

During day time reflection of sun light from the top of the cloud called albedo $(90 \%)$ dominates the green house effect of clouds which is only $50 \%$. And thus during day clouds have net cooling effect. So load consumption during a cloudy day will be low due to low temperature.

The cloud cover not only has impact on the temperature but also it can affect the light intensity at day time. If clouds are thick then it can block most of the sunlight, forcing the consumers to use electric bulbs etc even at the day time to light their houses, thus increasing the consumption of electricity. So for short term load forecasting we should consider not only the effect of cloud cover on temperature but also its effect on light intensity to forecast next hour / next day load more accurately.

\section{RANDOM OR OCCASIONAL SPIKES}

The power system consists of different types of consumers for example domestic, agricultural, industrial etc. The overall 
load of domestic consumers shows good statistical rules and is periodic in nature, but on the other hand industrial and agricultural loads are highly inductive and start up and shut down of such type of load induce huge spikes to the load curve. These spikes are called the random disturbance because start up and shut down of these huge loads is quite random in nature and there is no way to predict the occurrence of these spikes. CNG station load also lies in this category. If we add these spikes in the training data of ANN model then the average error of the model becomes very high [16].

Special events such as religious or cultural celebrations also are another source of random disturbance. Eid day, Christmas and other religious events such as Shab-e-Qadar are the examples of the special events.

Similarly Pakistan Vs India Cricket match also lies in the category of special days and is another source of random disturbance, causing a huge spike in the load curve due to increased usage of TV and cannot be accurately predicted because we do not know how much TV will be used during that day. Other typical events include strikes and government compulsory demand side management due to predicted shortfall of electricity.

\section{REFERENCES}

[1] Long-Term Hourly Peak Demand and Energy Forecast, Electric Reliability Council of Texas, Inc., Taylor, TX, 2010, pp. 9.

[2] S. Rahman, "Formulation and analysis of a rule-based short-term load forecasting algorithm," IEEE, vol. 78, pp. 805-816, 2002.

[3] M. Altalo and M. Hale, "Turning weather forecasts into business forecasts," Environmental Finance, May 2004.

[4] G. Franco and A. Sanstad, "Climate change and electricity demand in California," Climatic Change, vol. 87, pp. 139-151, 2007.

[5] D. Belzer, M. Scott, and R. Sands, "Climate change impacts on U.S. commercial building energy consumption, an analysis using sample survey data," Energy Sources, Part A: Recovery, Utilization, and Environmental Effects, vol. 18, pp. 177, 1996.

[6] D. Paravan, A. Debs, C. Hansen, D. Becker, P. Hirsch, and R. Golob. "Influence of temperature on short-term load forecasting using the EPRI-ANNSTLF."

[7] A. Pardo, V. Meneu, and E. Valor, "Temperature and seasonality influences on Spanish electricity load," Energy Economics, vol. 24, pp. 55-70, 2002.

[8] M. Bessec and J. Fouquau, "The non-linear link between electricity consumption and temperature in Europe: A threshold panel approach," Energy Economics, vol. 30, pp. 2705-2721, Sep. 2008.
[9] S. S. Wyer, "A treatise on producer-gas and gas-producers," The Engineering and Mining Journal, London, pp. 23, 1906.

[10] A. Azadeh, M. Saberi, and O. Seraj, "An integrated fuzzy regression algorithm for energy consumption estimation with non-stationary data: A case study of Iran," Energy, vol. 35, pp. 2351-2366, Jun 2010.

[11] A. Tajvidi et al., "An integrated simulated-based fuzzy regression algorithm and time series for energy consumption estimation with non-stationary data and case studies," presented at the IEEE International Conference on Digital Ecosystems and Technologies, 2009, pp. 139-143.

[12] H. G. Shakouri, R. Nadimi, and F. Ghaderi, "A hybrid TSK-FR model to study short-term variations of the electricity demand versus the temperature changes," Expert Systems with Applications, vol. 36, issue 2, pp. 1765-1772, March 2009.

[13] R. Zmeureanu and G. Renaud, "Estimation of potential impact of climate change on the heating energy use of existing houses," Energy Policy, vol. 36, pp. 303-310, Jan. 2008.

[14] W. Woodson et al., Human Factors Design Handbook, McGraw-Hill, 1981, pp. 815.

[15] R. Osczevski and M. Bluestein, "The new wind chill equivalent temperature chart," Bulletin of the American Meteorological Society, vol. 86, issue 10, pp. 1453-1458, Oct. 2005.

[16] D. K. Chaturvedi, S. A. Premdayal, and A. Chandiok, "Short-term load forecasting using soft computing techniques," Int. J. Communications, Network and System Sciences, vol. 3, no. 1, pp. 273-279, March 2010.

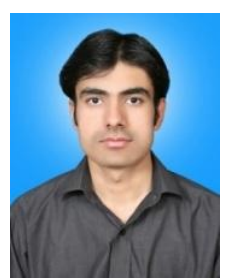

Muhammad Usman Fahad was born in Abbottabad, Pakistan. He graduated from the COMSATS University Pakistan in the field of Electronics Engineering in 2008. He is currently a student of Ms Electrical Engineering at the University of Engineering and Technology Peshawar in the field of Electrical Engineering (Power). He attended eight month course at the University of Bradford England and got the Certificate of Continuing Education in Electrical and Electronics engineering.

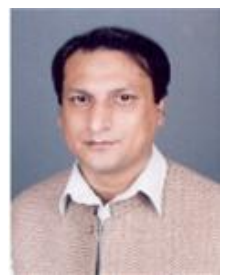

Muhammad Naeem Arbab received his MS and $\mathrm{PhD}$ degrees in 1985 and 1987 respectively from University of Manchester, U. K. His field of specialization is High Voltage Engineering. He has published several research papers in this field and has recently published a book on High Voltage Engineering. Presently he is serving as a professor in Electrical Engineering Department of University of Engineering and Technology, Peshawar, Pakistan. 